\title{
CONHECIMENTO DE ACADÊMICOS DE ENFERMAGEM SOBRE LESÕES POR PRESSÃO: DESAFIO PARA A SEGURANÇA DO PACIENTE
}

\author{
KNOWLEDGE OF NURSING STUDENTS ABOUT \\ PRESSURE INJURIES: CHALLENGE FOR \\ PATIENT SAFETY
}

\section{CONOCIMIENTO DE LOS ESTUDIANTES DE ENFERMERÍA ACERCA DE LAS LESIONES CAUSADAS POR PRESIÓN: DESAFÍO PARA LA SEGURIDAD DEL PACIENTE}

\author{
Ana Flávia Furtado ${ }^{1}$ \\ Larissa Marcondes ${ }^{2}$ \\ Bruna Eloise Lenhani ${ }^{3}$ \\ Josemar Batista ${ }^{4}$
}

\begin{abstract}
Como citar este artigo: Furtado AF, Marcondes L, Lenhani BE, Batista J. Conhecimento de acadêmicos de enfermagem sobre lesões por pressão: desafio para a segurança do paciente. Rev baiana enferm. 2019;33:e34425.

Objetivo: descrever o conhecimento dos acadêmicos de enfermagem em relação à avaliação, classificação e prevenção de lesões por pressão. Método: pesquisa transversal e quantitativa, realizada em instituição de ensino superior particular do estado do Paraná. Foi aplicado o questionário, Teste de Conhecimento de Pieper, no mês de maio de 2019, para 158 acadêmicos matriculados nos 2ํㅜ $3^{\circ}$ e $4^{\circ}$ anos do curso de graduação. Os dados foram analisados por estatística descritiva e analítica; questões com acertos $\geq 90 \%$ representaram conhecimento satisfatório. Resultados: o conhecimento sobre lesões por pressão foi considerado insatisfatório em 56,1\% das questões. A média de acertos dos acadêmicos do $4^{\circ}$ ano foi significativamente superior à média do $2^{\underline{o}}$ ano, para os itens relativos às medidas preventivas $(p=0,006)$. Nas demais comparações não houve diferenças significativas. Conclusão: o conhecimento dos acadêmicos de enfermagem com relação à avaliação, classificação e prevenção de lesões por pressão foi insatisfatório em todos os anos.
\end{abstract}

Descritores: Lesão por Pressão. Estudantes de Enfermagem. Conhecimento. Prevenção.

Objective: to describe the knowledge of nursing students in relation to evaluation, classification and prevention of pressure injuries. Method: cross-sectional and quantitative research, performed at a higher education institution in the state of Paraná. The questionnaire Pieper Knowledge Test was applied in May 2019 to 158 students enrolled in the second, third and fourth years of the graduate course. Data were analyzed through descriptive and analytical statistics; questions with $\geq 90 \%$ of right answers represented satisfactory knowledge. Results: the knowledge about

Estudante de Enfermagem. Faculdades Integradas Santa Cruz de Curitiba. Curitiba, Paraná, Brasil. https://orcid.org/0000-0002-1473-8482.

Enfermeira. Mestre em Enfermagem. Professora das Faculdades Integradas Santa Cruz de Curitiba. Curitiba, Paraná, Brasil. https://orcid.org/0000-0002-8745-6486.

Enfermeira. Mestre em Enfermagem. Professora das Faculdades Integradas Santa Cruz de Curitiba. Curitiba, Paraná, Brasil. https://orcid.org/0000-0002-6009-3400.

Enfermeiro. Mestre em Enfermagem. Professor das Faculdades Integradas Santa Cruz de Curitiba. Curitiba, Paraná, Brasil. josemar.batista@hotmail.com https://orcid.org/0000-000 I-9838-1232. 
pressure injuries was considered unsatisfactory in $56.1 \%$ of the questions. The mean scores of students from the fourth year was significantly higher than the average of the second year for items concerning preventive measures $(p=0.006)$. In other comparisons, there were no significant differences. Conclusion: the knowledge of the nursing students with respect to the evaluation, classification and prevention of pressure injuries was unsatisfactory in all years.

Descriptors: Pressure Ulcer. Students, Nursing. Knowledge. Prevention.

Objetivo: describir el conocimiento de los estudiantes de enfermería en relación con la evaluación, clasificación y prevención de las lesiones causadasporpresión. Método: estudio transversaly cuantitativo, realizadoen una institución de educación superior en el estado de Paraná. El cuestionario Test de Conocimientos de Pieper fue aplicado, en el mes de mayo de 2019, a 158 estudiantes matriculados en el $2^{\circ}, 3^{\circ}$ y $4^{\circ}$ años del curso universitario. Los datos fueron analizados mediante estadística descriptiva y analítica; cuestiones con éxitos $\geq 90 \%$ representaron un conocimiento satisfactorio. Resultados: el conocimiento acerca de las lesiones causadas por presión fue considerado insatisfactorio en el 56,1\% de las cuestiones. Las puntuaciones medias de los alumnos del $4^{\circ}$ año fue significativamente mayor que el promedio del segundo año para artículos sobre medidas preventivas $(p=0,006)$. En otras comparaciones, no hubo diferencias significativas. Conclusión: el conocimiento de los estudiantes de enfermería con respecto a la evaluación, clasificación y prevención de lesiones causadas por la presión fue insatisfactorio en todos los años.

Descriptores: Úlcera por Presión. Estudiantes de Enfermería. Conocimiento. Prevención.

\section{Introdução}

A prevenção, o tratamento e a reabilitação de indivíduos com lesões de pele constituem atribuições dos profissionais de saúde, em especial do enfermeiro ${ }^{(1)}$. Entretanto, percebe-se que essas ações continuam desafiadoras para os serviços de saúde, principalmente no que se refere às lesões por pressão (LPP), em virtude de sua etiologia multifatorial e da dinamicidade dos fatores predisponentes (intrínsecos e extrínsecos) que contribuem para o desenvolvimento desse tipo de lesão ${ }^{(2-3)}$.

A LPP é definida pelo National Pressure Ulcer Advisory Panel (NPUAP) como qualquer dano localizado na pele e/ou tecidos subjacentes, geralmente sobre proeminência óssea, decorrente de intensa e/ou prolongada pressão ou de pressão combinada ao cisalhamento ${ }^{(4-5)}$. Estão classificadas em estágio I (pele íntegra com eritema que não embranquece), estágio II (perda parcial da espessura da pele com exposição dessa), estágio III (perda total da espessura da pele), estágio IV (perda total da espessura da pele e perda tissular), e não classificáveis (lesão tissular profunda, lesão relacionada ao uso de dispositivos médicos e lesão em membrana mucosa) ${ }^{(4-5)}$.

A LPP e a sua prevenção são consideradas importantes indicadores de qualidade e são classificadas em eventos adversos (EA) evitáveis ${ }^{(2)}$. Estes impactam na vida do indivíduo que a desenvolve, na de seus familiares e na instituição $^{(4)}$, com ascensão no tempo de internação, da mortalidade e dos custos hospitalares ${ }^{(6)}$. Apesar dos esforços contínuos dos profissionais de saúde, em especial da enfermagem, no que se refere ao desenvolvimento de ações em prol da prevenção de LPP, observa-se elevada prevalência e incidência desse EA no ambiente hospitalar. Em estudo multicêntrico, realizado em 25 hospitais da China, a prevalência foi de 3,38\%; 84,03\% foram classificadas em estágios I e II. As regiões sacral e calcanhar foram as mais acometidas $(48,22 \%)^{(7)}$.

Quanto ao Brasil, segundo o Relatório Nacional de Incidentes Relacionados à Assistência à Saúde, no período de março de 2014 a maio de 2019, dos 325.430 casos notificados, 59.417 $(18,3 \%)$ corresponderam às notificações LPP, sendo, durante esse período, o terceiro tipo de evento mais notificado pelos estabelecimentos de saúde do país. O referido relatório realçou a ocorrência de, aproximadamente, 8.245 never events (eventos que nunca deveriam ocorrer em serviços de saúde), sendo 5.946 (72,1\%) decorrentes de LPP, estágio III, e 1.807 (21,9\%) 
resultantes de LPP, estágio IV. Quanto aos óbitos notificados nesse período, cerca de 49 (3\%) foram ocasionados por esse $\mathrm{EA}^{(8)}$.

Frente ao exposto, observa-se que as LPP persistem como um problema relevante e atual, no contexto da segurança do paciente, nas organizações de saúde em âmbito mundial e nacional. Desta forma, diretrizes relacionadas à avaliação, diagnóstico, prevenção e tratamento de LPP são preconizadas por órgãos internacionais, tais como o National Pressure Ulcer Advisory Panel (NPUAP), o European Pressure Ulcer Advisory Panel (EPUAP), a Pan Pacific Pressure Injury Alliance (PPPIA) e o National Institute for Health and Care Excellence (NICE), com vistas a direcionar a prática clínica ${ }^{(9-10)}$.

A implementação de ações para reduzir os casos de LPP também é recomendada pelo Programa Nacional de Segurança do Paciente (PNSP) e torna-se fundamental para qualificar o cuidado em todos os estabelecimentos de saúde do território brasileiro ${ }^{(11)}$. O referido programa tem um eixo específico para prevenção e tratamento de LPP. Desse modo, aliar o aumento do conhecimento por parte da equipe de enfermagem à implementação de práticas baseadas em evidências resulta em benefícios tanto na redução do tempo de internação hospitalar quanto no número de pacientes acometidos por esse agravo ${ }^{(6,12)}$.

Nesse contexto, acredita-se que, para a implementação de boas práticas assistenciais, com base nos preceitos do protocolo básico de segurança relacionado à prevenção dessas lesões, parte-se do princípio de que o conhecimento adquirido pelo profissional enfermeiro, durante a formação acadêmica, torna-se essencial para operacionalizar estratégias que reduzam esses eventos em serviços públicos e privados do país.

Partindo do pressuposto de que investigar o conhecimento dos acadêmicos de enfermagem pode contribuir na identificação de lacunas oriundas do processo de formação profissional e subsidiar ações educativas, visando atender às diretrizes do PNSP, questiona-se: Qual é o nível de conhecimento de acadêmicos de enfermagem referente à avaliação, classificação e medidas preventivas em pacientes com lesões por pressão?

O objetivo deste estudo é descrever o conhecimento dos acadêmicos de enfermagem em relação à avaliação, classificação e prevenção de lesões por pressão.

\section{Método}

Trata-se de uma pesquisa transversal, com abordagem quantitativa, realizado em uma instituição de ensino superior particular, no estado do Paraná. A população foi composta por todos os acadêmicos do curso de graduação em enfermagem, devidamente matriculados nos $2^{\circ}$, $3^{\circ}$ e $4^{\underline{0}}$ anos do curso, totalizando 158 acadêmicos. Desses, 42 cursavam o segundo ano de graduação, 75 o terceiro e 41 o último ano ( $4^{\text {o }}$ ano). Para se obter uma amostra com 95\% de confiança, margem de erro igual a 5\% e proporção de prevalência conservadora igual a 0,5 , calculou-se a amostragem estratificada proporcional, baseada na proporção da população de acadêmicos de cada ano. O resultado desse cálculo totalizou 114 acadêmicos.

Foram critérios de inclusão: ser acadêmico de enfermagem e aprovado ou cursando a disciplina de Semiologia e Métodos Assistenciais, ofertada no primeiro semestre do segundo ano (3o período), da grade curricular do curso de graduação em enfermagem. Cabe destacar que o conteúdo contemplado pelo objeto de estudo desta pesquisa foi ministrado em aulas expositivas e habilidades práticas em laboratório no semestre. Foram excluídos acadêmicos com idade inferior a 18 anos e aqueles que devolveram o questionário em branco e/ou tiveram menos de 50\% das perguntas preenchidas. Após aplicação desses critérios, nenhum participante foi excluído da análise.

Os acadêmicos foram recrutados por conveniência, em sala de aula, no horário do intervalo (matutino e noturno). A participação foi condicionada ao prévio consentimento do acadêmico e à assinatura do Termo de Consentimento Livre e Esclarecido, com as devidas orientações e esclarecimentos pertinentes à pesquisa. A coleta 
de dados ocorreu no mês de maio de 2019, em sala reservada, mediante a entrega de envelope lacrado, contendo o questionário denominado Teste de Conhecimento de Pieper ${ }^{(13)}$, traduzido e validado para o Brasil ${ }^{(14)}$.

O questionário autoaplicável continha 41 itens, com questões cujas respostas atendiam ao modelo verdadeiro, falso e não sei, divididas em categoria 1 (Avaliação e Classificação da LPP), com 8 itens, e Categoria 2, com 33 questões sobre medidas preventivas de LPP. Cada resposta correta (V ou F) valia um ponto. A pontuação superior ou igual a 37 pontos (90\%) foi considerada satisfatória ${ }^{(13)}$. Os envelopes foram recolhidos pelos pesquisadores e codificados com letra e numeral para designar o ano do curso (A2, A3 e A4), seguido pela numeração sequencial dos respondentes de cada ano investigado (P1, P2, ..P30), garantindo-se o anonimato dos participantes.

Os dados foram transcritos em planilhas, no programa Microsoft Office Excel 2016, por dupla digitação e correção de inconsistências. Após a exclusão dos itens deixados em branco no questionário, as variáveis quantitativas foram descritas por média e desvio padrão; as variáveis qualitativas foram apresentadas em frequências absoluta e relativa. Para avaliar se as respostas às questões (acertos) estavam associadas ao ano de curso do acadêmico, foi utilizado o teste Qui-quadrado e o Kruskall-Wallis. Para identificar quais grupos, de fato, diferiram significativamente, foi necessário realizar comparações múltiplas de todas as categorias, pelo teste post-hoc de Dunn, com correção do valor-p, do tipo False Discovery Rate. Os dados foram processados pelo software R e assessoria estatística; o nível de significância adotado foi de 5\% ( $<<0,05)$.

A pesquisa foi aprovada pelo Comitê de Ética em Pesquisa em Seres Humanos institucional sob Parecer n. 3.305.836.

\section{Resultados}

A idade média dos acadêmicos foi de 27,87 anos, com variação entre 18 e 50 anos (desvio padrão de 8,57$)$. Houve prevalência de participantes do sexo feminino $(80,7 \% ; n=92)$ e sem experiência na área da saúde $(52,6 \% ; n=60)$.

A Tabela 1 mostra o conhecimento dos acadêmicos de enfermagem, no que se refere à avaliação e à classificação das LPP. Seis questões foram consideradas insatisfatórias (pontuação $\leq 90 \%$ de acertos); entretanto, houve diferença significativa no percentual de acertos, entre os acadêmicos do $2^{\circ}$ e $4^{\underline{o}}$ ano $(\mathrm{p}<0,001)$.

Tabela 1 - Distribuição das respostas dos acadêmicos de enfermagem, conforme o conhecimento referente à avaliação e à classificação das lesões por pressão. Curitiba, Paraná, Brasil - 2019 (continua)

\begin{tabular}{|c|c|c|c|c|c|}
\hline \multirow{2}{*}{ Avaliação e classificação das lesões por pressão } & \multirow{2}{*}{ Ano } & \multicolumn{3}{|c|}{$\begin{array}{c}\text { Percentual de } \\
\text { respostas* }\end{array}$} & \multirow{2}{*}{ Valor $\mathbf{p}^{* *}$} \\
\hline & & Certas & Erradas & $\begin{array}{l}\text { Não } \\
\text { sei }\end{array}$ & \\
\hline $\begin{array}{l}\text { 1. O estágio I da úlcera (lesão) por pressão é definido } \\
\text { como pele intacta, com hiperemia de uma área } \\
\text { localizada, a qual não apresenta embranquecimento } \\
\text { visível ou a cor difere da área ao redor (V) }\end{array}$ & $\begin{array}{l}2^{\mathrm{O}} \\
3^{\mathrm{O}} \\
4^{\mathrm{O}}\end{array}$ & $\begin{array}{l}60,0 \\
66,0 \\
66,7\end{array}$ & $\begin{array}{l}40,0 \\
24,5 \\
30,0\end{array}$ & $\begin{array}{c}- \\
9,4 \\
3,3\end{array}$ & 0,270 \\
\hline $\begin{array}{l}\text { 6. Uma úlcera (lesão) por pressão, em estágio III, é } \\
\text { perda parcial de pele, envolvendo a epiderme (F) }\end{array}$ & $\begin{array}{l}2^{\mathrm{o}} \\
3^{-} \\
4^{\mathrm{o}}\end{array}$ & $\begin{array}{l}26,7 \\
13,5 \\
14,3\end{array}$ & $\begin{array}{l}70,0 \\
78,8 \\
82,1\end{array}$ & $\begin{array}{l}3,3 \\
7,7 \\
3,6\end{array}$ & 00,504 \\
\hline $\begin{array}{l}\text { 9. As úlceras (lesões) por pressão, no estágio } \\
\text { IV, apresentam perda total de pele com intensa } \\
\text { destruição e necrose tissular ou danos aos músculos, } \\
\text { ossos ou estruturas de suporte (V) }\end{array}$ & $\begin{array}{l}2^{\mathrm{O}} \\
3^{\mathrm{O}} \\
4^{\mathrm{O}}\end{array}$ & $\begin{array}{l}90,0 \\
87,0 \\
93,3\end{array}$ & $\begin{array}{c}3,3 \\
- \\
3,3\end{array}$ & $\begin{array}{c}6,7 \\
13,0 \\
3,3\end{array}$ & 00,389 \\
\hline $\begin{array}{l}\text { 20. As úlceras (lesões) por pressão, no estágio II, } \\
\text { apresentam perda de pele em sua espessura total (F) }\end{array}$ & $\begin{array}{l}2^{-} \\
3^{-} \\
4^{-}\end{array}$ & $\begin{array}{l}48,3 \\
34,6 \\
63,3\end{array}$ & $\begin{array}{l}41,4 \\
44,2 \\
33,3\end{array}$ & $\begin{array}{c}10,3 \\
21,2 \\
3,3\end{array}$ & 00,067 \\
\hline
\end{tabular}


Tabela 1 - Distribuição das respostas dos acadêmicos de enfermagem, conforme o conhecimento referente à avaliação e à classificação das lesões por pressão. Curitiba, Paraná, Brasil - 2019 (conclusão)

\begin{tabular}{|c|c|c|c|c|c|}
\hline \multirow{2}{*}{ Avaliação e classificação das lesões por pressão } & \multirow{2}{*}{ Ano } & \multicolumn{3}{|c|}{$\begin{array}{c}\text { Percentual de } \\
\text { respostas* }\end{array}$} & \multirow{2}{*}{ Valor $\mathbf{p}^{* *}$} \\
\hline & & Certas & Erradas & $\begin{array}{l}\text { Não } \\
\text { sei }\end{array}$ & \\
\hline \multirow{3}{*}{$\begin{array}{l}\text { 31. As úlceras (lesões) por pressão são feridas estéreis } \\
\text { (F) }\end{array}$} & $2^{\mathrm{O}}$ & 69,0 & 6,9 & 24,1 & \multirow{3}{*}{00,064} \\
\hline & $3^{\mathrm{o}}$ & 54,9 & 25,5 & 19,6 & \\
\hline & $4^{-}$ & 80,0 & 13,3 & 6,7 & \\
\hline \multirow{3}{*}{$\begin{array}{l}\text { 32. Uma região da pele com cicatriz da úlcera (lesão) } \\
\text { por pressão poderá ser lesada mais rapidamente do } \\
\text { que a pele íntegra (V) }\end{array}$} & $2^{\mathrm{O}}$ & 53,6 & 17,9 & 28,6 & \multirow[t]{3}{*}{00,208} \\
\hline & $3^{\mathrm{o}}$ & 75,5 & 9,4 & 15,1 & \\
\hline & $4^{\mathrm{o}}$ & 80,0 & 6,7 & 13,3 & \\
\hline \multirow{3}{*}{$\begin{array}{l}\text { 33. Uma bolha na região do calcâneo não deve ser } \\
\text { motivo para preocupação }(\mathrm{F})\end{array}$} & $2^{\underline{\mathrm{o}}}$ & 62,1 & 13,8 & 24,1 & \multirow[t]{3}{*}{$<0,001$} \\
\hline & $3^{\mathrm{o}}$ & 87,0 & 11,1 & 1,9 & \\
\hline & $4^{\mathrm{O}}$ & 96,7 & 3,3 & - & \\
\hline \multirow{3}{*}{$\begin{array}{l}\text { 38. As úlceras (lesões) por pressão, de estágio II, } \\
\text { podem ser extremamente doloridas, em decorrência } \\
\text { da exposição das terminações nervosas (V) }\end{array}$} & $2^{\mathrm{o}}$ & 75,9 & 10,3 & 13,8 & \multirow[t]{3}{*}{$<0,001$} \\
\hline & $3^{\circ}$ & 61,1 & 20,4 & 18,5 & \\
\hline & $4^{\mathrm{o}}$ & 33,3 & 56,7 & 10,0 & \\
\hline
\end{tabular}

Fonte: Elaboração própria.

Nota: Sinal convencional utilizado:

- Dado numérico igual a zero não resultante de arredondamento.

* $2^{\mathrm{o}}$ ano $(\mathrm{n}=42) ; 3^{\circ}$ ano $(\mathrm{n}=75) ; 4^{\circ}$ ano $(\mathrm{n}=41)$.

** Teste Qui-quadrado $\mathrm{p}<0,05$.

A depender do ano analisado, a Tabela 2 mostra que 16 questões obtiveram índice de acertos igual ou superior a $90 \%$. Os acadêmicos do $4^{\circ}$ ano externaram significativamente conhecimento mais satisfatório em três questões $(p<0,05)$, a saber: "Todos os pacientes em risco para úlcera (lesão) por pressão devem ter inspeção sistemática da pele pelo menos uma vez por semana", "As regiões das proeminências ósseas podem ficar em contato direto uma com a outra" e "Os pacientes hospitalizados necessitam ser avaliados quanto ao risco para úlcera (lesão) por pressão uma única vez durante sua internação".

Tabela 2 - Distribuição das respostas dos acadêmicos de enfermagem, conforme o conhecimento das medidas de prevenção da lesão por pressão. Curitiba, Paraná, Brasil - 2019

(continua)

\begin{tabular}{|c|c|c|c|c|c|}
\hline \multirow{2}{*}{ Medidas de prevenção da lesão por pressão } & \multirow{2}{*}{ Ano } & \multicolumn{3}{|c|}{ Percentual de respostas* } & \multirow{2}{*}{ Valor $\mathbf{p}^{* *}$} \\
\hline & & Certas & Erradas & Não sei & \\
\hline $\begin{array}{l}\text { 2. Os fatores de risco para o desenvolvimento } \\
\text { da úlcera (lesão) por pressão são: imobilidade, } \\
\text { incontinência, nutrição inadequada e alteração } \\
\text { do nível de consciência (V) }\end{array}$ & $\begin{array}{l}2^{\circ} \\
3^{\circ} \\
4^{\circ}\end{array}$ & $\begin{array}{l}70,0 \\
67,3 \\
63,3\end{array}$ & $\begin{array}{l}23,3 \\
28,8 \\
29,5\end{array}$ & $\begin{array}{c}6,7 \\
36,7 \\
-\end{array}$ & 0,574 \\
\hline $\begin{array}{l}\text { 3. Todos os pacientes em risco para úlcera } \\
\text { (lesão) por pressão devem ter inspeção } \\
\text { sistemática da pele pelo menos uma vez por } \\
\text { semana (F) }\end{array}$ & $\begin{array}{l}2^{\mathrm{o}} \\
3^{\mathrm{O}} \\
4^{\mathrm{O}}\end{array}$ & $\begin{array}{l}34,5 \\
50,9 \\
76,7\end{array}$ & $\begin{array}{l}55,2 \\
47,2 \\
23,3\end{array}$ & $\begin{array}{c}10,3 \\
1,9 \\
-\end{array}$ & 0,007 \\
\hline $\begin{array}{l}\text { 4. O uso de água quente e sabonete pode } \\
\text { ressecar a pele e aumentar o risco para úlcera } \\
\text { (lesão) por pressão (V) }\end{array}$ & $\begin{array}{l}2^{\underline{0}} \\
3^{-} \\
4^{-}\end{array}$ & $\begin{array}{l}40,0 \\
51,9 \\
63,3\end{array}$ & $\begin{array}{l}30,0 \\
35,2 \\
26,7\end{array}$ & $\begin{array}{l}30,0 \\
13,0 \\
10,0\end{array}$ & 0,165 \\
\hline $\begin{array}{l}\text { 5. É importante massagear as regiões } \\
\text { das proeminências ósseas, se estiverem } \\
\text { hiperemiadas (F) }\end{array}$ & $\begin{array}{l}2^{\underline{0}} \\
3^{\circ} \\
4^{\underline{o}}\end{array}$ & $\begin{array}{l}24,1 \\
36,5 \\
31,0\end{array}$ & $\begin{array}{l}55,2 \\
50,0 \\
48,3\end{array}$ & $\begin{array}{l}20,7 \\
13,5 \\
20,7\end{array}$ & 0,756 \\
\hline
\end{tabular}


Tabela 2 - Distribuição das respostas dos acadêmicos de enfermagem, conforme o conhecimento das medidas de prevenção da lesão por pressão. Curitiba, Paraná, Brasil - 2019

Medidas de prevenção da lesão por pressão

7. Todos os pacientes devem ser avaliados na sua admissão no hospital, quanto ao risco para desenvolvimento da úlcera (lesão) por pressão (V)

8. Os cremes, curativos transparentes e curativos de hidrocoloides extrafinos auxiliam na proteção da pele contra os efeitos da fricção (V)

10. Uma ingestão dietética adequada de proteínas e calorias deve ser mantida durante a doença/hospitalização (V)

11. Os pacientes que ficam restritos ao leito devem ser reposicionados a cada 3 horas (F)

12. Uma escala com horários para mudança de decúbito deve ser utilizada para cada paciente com presença ou em risco para úlcera (lesão) por pressão (V)

13. As luvas d'água ou de ar aliviam a pressão nos calcâneo $(\mathrm{F})$

14. As almofadas tipo rodas d'água ou de ar auxiliam na prevenção da úlcera (lesão) por pressão (F)

15. Na posição em decúbito lateral, o paciente com presença da úlcera (lesão) por pressão ou em risco para esta deve ficar em ângulo de 30 graus em relação ao colchão do leito (V)

16. No paciente com presença da úlcera (lesão) por pressão ou em risco para esta, a cabeceira da cama não deve ser elevada em ângulo maior do que 30 graus, se não houver contraindicação médica (V)

17. O paciente que não se movimenta sozinho deve ser reposicionado a cada 2 horas, quando sentado na cadeira $(F)$

18. O paciente com mobilidade limitada e que pode mudar a posição do corpo sem ajuda, deve ser orientado a realizar o alívio da pressão, a cada 15 minutos, enquanto estiver sentado na cadeira (V)

19. O paciente com mobilidade limitada, e que pode permanecer na cadeira, deve ter uma almofada no assento para proteção da região das proeminências ósseas (V)

21. A pele do paciente em risco para úlcera (lesão) por pressão, deve permanecer limpa e livre de umidade (V)

\section{Ano}

Percentual de respostas*

Certas $\quad$ Erradas $\quad$ Não sei

$\begin{array}{lllll}2^{\mathrm{o}} & 90,0 & 3,3 & 6,7 & 0,434\end{array}$

$3^{\text {ㅇ }} \quad 88,7 \quad 9,4 \quad 1,9$

93,3

6,7

\section{3,3}

10,0

$-$

$3^{\circ}$

$\begin{array}{llll}4^{\circ} & 86,7 & 10,0 & 3,3\end{array}$

$4^{\circ} \quad 90,0 \quad 10,0$


Tabela 2 - Distribuição das respostas dos acadêmicos de enfermagem, conforme o conhecimento das medidas de prevenção da lesão por pressão. Curitiba, Paraná, Brasil - 2019

\section{Medidas de prevenção da lesão por pressão}

As medidas para prevenir novas lesões não necessitam ser adotadas continuamente quando o paciente já possui úlcera (lesão) por pressão (F)

23. Os lençóis móveis ou forros devem ser utilizados para transferir ou movimentar pacientes que não se movimentam sozinhos (V)

24. A mobilização e a transferência de pacientes que não se movimentam sozinhos devem ser sempre realizadas por duas ou mais pessoas (V)

25. No paciente com condição crônica que não se movimenta sozinho, a reabilitação deve ser iniciada e incluir orientações sobre a prevenção e o tratamento da úlcera (lesão) por pressão (V)

26. Todo paciente que não deambula deve ser submetido à avaliação de risco para o desenvolvimento da úlcera (lesão) por pressão (V)

27. Os pacientes e familiares devem ser orientados quanto às causas e aos fatores de risco para o desenvolvimento da úlcera (lesão) por pressão (V)

28. As regiões das proeminências ósseas podem ficar em contato direto uma com a outra (F)

29. Todo paciente em risco para desenvolver úlcera (lesão) por pressão deve ter um colchão que redistribua a pressão (V)

30. A pele, quando macerada pela umidade, danifica-se mais facilmente (V)

34. Uma boa maneira de diminuir a pressão na região dos calcâneos é mantê-los elevados do leito (V)

35. Todo cuidado para prevenir ou tratar úlceras (lesões) por pressão não precisa ser registrado (F)

36. Cisalhamento é a força que ocorre quando a pele adere a uma superfície e o corpo desliza (V)

37. A fricção pode ocorrer ao se movimentar o paciente sobre o leito (V)

39. No paciente com incontinência, a pele deve ser limpa no momento das eliminações e nos intervalos de rotina (V)

\begin{tabular}{|c|c|c|c|c|}
\hline \multirow{2}{*}{ Ano } & \multicolumn{3}{|c|}{ Percentual de respostas* } & \multirow{2}{*}{ Valor $\mathbf{p}^{* *}$} \\
\hline & Certas & Erradas & Não sei & \\
\hline $2^{\underline{o}}$ & 75,9 & 20,7 & 3,4 & 0,491 \\
\hline $3^{\mathrm{o}}$ & 79,2 & 13,2 & 7,5 & \\
\hline $4^{\mathrm{o}}$ & 80,0 & 20,0 & - & \\
\hline $2^{\underline{o}}$ & 89,7 & 10,3 & - & 0,215 \\
\hline $3^{\circ}$ & 77,4 & 17,0 & 17,0 & \\
\hline
\end{tabular}

$\begin{array}{lll}4^{\circ}-93,3 & 6,7\end{array}$

$2^{\mathrm{o}} \quad 96,6 \quad 3,4$

3ㅇ $\quad 94,4 \quad 1,9 \quad 3,7$

$\begin{array}{llll}4^{\circ} & 96,7 & 3,3\end{array}$

$2^{\mathrm{o}} \quad 78,6 \quad 10,7 \quad 10,7$

3은 $\quad 88,7 \quad 3,8 \quad 3,8$

$4^{\mathrm{O}} \quad 100,0$
$3^{\mathrm{O}}$

100,0 
Tabela 2 - Distribuição das respostas dos acadêmicos de enfermagem, conforme o conhecimento das medidas de prevenção da lesão por pressão. Curitiba, Paraná, Brasil - 2019

(conclusão)

\begin{tabular}{|c|c|c|c|c|c|}
\hline \multirow{2}{*}{ Medidas de prevenção da lesão por pressão } & \multirow{2}{*}{ Ano } & \multicolumn{3}{|c|}{ Percentual de respostas* } & \multirow{2}{*}{ Valor $\mathbf{p}^{* *}$} \\
\hline & & Certas & Erradas & Não sei & \\
\hline \multirow{3}{*}{$\begin{array}{l}\text { 40. O desenvolvimento de programas } \\
\text { educacionais na instituição pode reduzir a } \\
\text { incidência da úlcera (lesão) por pressão (V) }\end{array}$} & $2^{\underline{o}}$ & 89,7 & 3,4 & 6,9 & \multirow[t]{3}{*}{0,376} \\
\hline & $3^{\circ}$ & 87,0 & 5,6 & - & \\
\hline & $4^{\underline{0}}$ & 100,0 & - & - & \\
\hline \multirow{3}{*}{$\begin{array}{l}\text { 41. Os pacientes hospitalizados necessitam ser } \\
\text { avaliados quanto ao risco para úlcera (lesão) por } \\
\text { pressão uma única vez durante sua internação (F) }\end{array}$} & $2^{\mathrm{o}}$ & 62,1 & 31,0 & 6,9 & \multirow[t]{3}{*}{0,029} \\
\hline & $3^{\circ}$ & 70,4 & 24,1 & 5,6 & \\
\hline & $4^{-}$ & 96,7 & 3,3 & - & \\
\hline
\end{tabular}

Fonte: Elaboração própria.

Nota: Sinal convencional utilizado:

- Dado numérico igual a zero não resultante de arredondamento.

* $2^{\mathrm{o}}$ ano $(\mathrm{n}=42) 3^{\circ}$ ano $(\mathrm{n}=75) 4^{\mathrm{o}}$ ano $(\mathrm{n}=41)$.

* Teste de Qui-quadrado, $\mathrm{p}<0,05$.

A Figura 1 e a Tabela 3 mostram o número/média de acertos, de acordo com a categoria de conhecimentos, em relação ao ano do curso. Observa-se que, para a categoria 2 (Medidas preventivas de LPP), o valor do teste de Dunn apontou que a média de acertos dos acadêmicos do último ano foi significativamente superior à média do $2^{\circ}$ ano $(\mathrm{p}=0,006)$. Nas demais comparações não houve diferenças significativas.

Figura 1 - Distribuição do número de acertos dos acadêmicos de enfermagem para cada categoria, em relação ao ano do curso de graduação
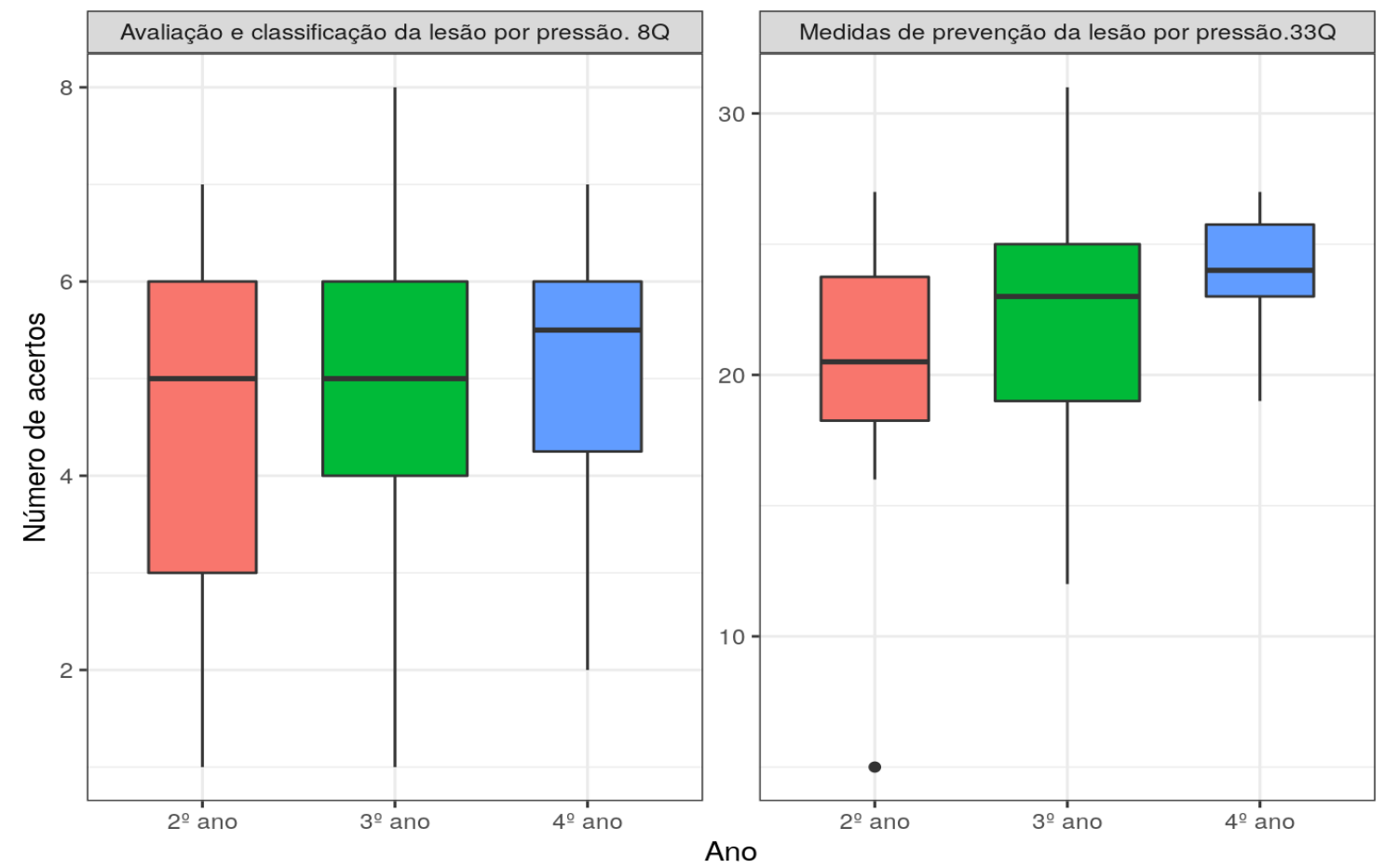

Fonte: Elaboração própria. 
Tabela 3 - Distribuição das medidas descritivas e valor-p para as diferenças de médias/pontos, segundo a categoria de conhecimentos dos acadêmicos de enfermagem do 2º ano. Curitiba, Paraná, Brasil - 2019

\begin{tabular}{|c|c|c|c|c|c|c|}
\hline \multirow[b]{2}{*}{ Variáveis } & \multicolumn{5}{|c|}{$2^{o}$ ano $(n=30)$} & \multirow[b]{2}{*}{ Valor-p* } \\
\hline & mínimo & média & mediana & $\begin{array}{l}\text { Desvio } \\
\text { padrão }\end{array}$ & máximo & \\
\hline $\begin{array}{l}\text { Avaliação e classificação da lesão } \\
\text { por pressão }\end{array}$ & 1 & 5 & 5 & 2 & 7 & 0,221 \\
\hline $\begin{array}{l}\text { Medidas preventivas da lesão por } \\
\text { pressão }\end{array}$ & 5 & 21 & 20 & 4 & 27 & 0,009 \\
\hline
\end{tabular}

\begin{tabular}{|c|c|c|c|c|c|c|}
\hline \multirow[b]{2}{*}{ Variáveis } & \multicolumn{5}{|c|}{$3^{\circ}$ ano $(n=30)$} & \multirow[b]{2}{*}{ Valor-p* } \\
\hline & mínimo & média & mediana & $\begin{array}{l}\text { Desvio } \\
\text { padrão }\end{array}$ & máximo & \\
\hline $\begin{array}{l}\text { Avaliação e classificação da lesão } \\
\text { por pressão }\end{array}$ & 1 & 5 & 5 & 1 & 8 & 0,221 \\
\hline $\begin{array}{l}\text { Medidas preventivas da lesão por } \\
\text { pressão }\end{array}$ & 12 & 22 & 23 & 4 & 31 & 0,009 \\
\hline
\end{tabular}

\begin{tabular}{|c|c|c|c|c|c|c|}
\hline \multirow[b]{2}{*}{ Variáveis } & \multicolumn{5}{|c|}{$4^{\circ}$ ano $(n=30)$} & \multirow[b]{2}{*}{ Valor-p* } \\
\hline & mínimo & média & mediana & $\begin{array}{l}\text { Desvio } \\
\text { padrão }\end{array}$ & máximo & \\
\hline $\begin{array}{l}\text { Avaliação e classificação da lesão } \\
\text { por pressão }\end{array}$ & 2 & 5 & 6 & 1 & 7 & 0,221 \\
\hline $\begin{array}{l}\text { Medidas preventivas da lesão } \\
\text { por pressão }\end{array}$ & 19 & 24 & 24 & 2 & 27 & 0,009 \\
\hline
\end{tabular}

Fonte: Elaboração própria.

* Teste de Kruskal-Wallis, $\mathrm{p}<0,05$.

\section{Discussão}

$\mathrm{Na}$ amostra da presente pesquisa, observa-se que a busca pelo curso de graduação em enfermagem continua predominantemente feminina e composta por adultos e jovens. Esses dados confirmam o perfil de acadêmicos de outras instituições de ensino superior do Brasil, encontrado em pesquisas que apontam o percentual aproximado de 20\% dos acadêmicos matriculados do sexo masculino ${ }^{(15-16)}$.

Com vistas a fornecer cuidados de excelência por enfermeiros, no que se refere à avaliação, classificação e medidas preventivas para LPP, é sabido que avaliar o processo de ensino e aprendizagem na temática, em instituições de ensino, torna-se relevante para propor ações de melhoria na formação de futuros profissionais de enfermagem, bem como na construção do conhecimento. Ao atender aos preceitos de prevenção e tratamento de LPP estabelecidos pelo
PNSP, também se almejam melhorias contínuas da prática clínica da enfermagem ${ }^{(11)}$.

Ocasionalmente, em estudo prévio realizado em universidade pública brasileira, com o objetivo de verificar a compreensão de 15 acadêmicos de Enfermagem sobre a segurança do paciente, identificou-se que associaram as suas ações às metas do referido programa, com exceção da prevenção de $\operatorname{LPP}^{(17)}$. Isso explica, em parte, os dados aqui apresentados, ao revelar que o conhecimento dos acadêmicos foi insuficiente, ao considerar que mais da metade das questões obtiveram escore de acertos inferior a 90\%, independentemente do ano investigado.

Ao considerar que a prevenção e o tratamento de LPP são metas relacionadas à segurança do paciente, esses achados são preocupantes, diante da necessidade de operacionalização das seis etapas propostas pelo protocolo ministerial de prevenção de LPP, a saber: avaliação de LPP na admissão de todos os pacientes; reavaliação 
10

Conhecimento de acadêmicos de enfermagem sobre lesões por pressão: desafio para a segurança do paciente

diária de risco de desenvolvimento de LPP de todos os pacientes internados; inspeção diária da pele; manejo da umidade; otimização da nutrição e da hidratação; e utilização de recursos redutores de pressão(6). Além do mais, essa circunstância potencializa a subnotificação de LPP, ao mesmo tempo que limita os acadêmicos, enquanto futuros gestores, a apoiarem a promoção de uma cultura de segurança nas instituições de saúde, que incentiva a aprendizagem em torno das falhas e institui medidas de prevenção desses eventos ${ }^{(12)}$.

Estudos brasileiros realizados com profissionais de enfermagem, com tempo de atuação profissional majoritariamente superior a três anos, confirmaram a insuficiência de conhecimento sobre o tema abordado ${ }^{(18-19)}$. Frente a este panorama, é possível perceber e reconhecer que o conhecimento relacionado aos 41 itens abordados no questionário é limitante não só entre acadêmicos de enfermagem como também entre profissionais formados. Esse fato é preocupante e representa um fator desfavorável na oferta de assistência de enfermagem livre de danos à pessoa com lesões de pele, pois o conhecimento desses profissionais é um demonstrativo de como esses cuidados estão sendo gerenciados e realizados na prática ${ }^{(20)}$.

Na categoria 1 (Avaliação e Classificação das LLP) foi constatado que apenas duas questões obtiveram $90 \%$ ou mais de acertos entre os acadêmicos do $2^{\circ}$ e $4^{\circ}$ ano. Esse achado é semelhante ao descrito em investigação conduzida em uma universidade pública brasileira, com 23 acadêmicos de enfermagem do último ano, a qual apontou que apenas um acadêmico teve $90 \%$ de acertos ${ }^{(21)}$. Outra investigação, conduzida com 56 acadêmicos de enfermagem de duas instituições de ensino situadas na região Nordeste brasileira, apontou que o número de acertos referente aos itens avaliação e classificação da LPP foi de $33,3 \%{ }^{(16)}$.

Nota-se que apenas uma questão (item 33) apresentou diferença significativa entre o conhecimento dos acadêmicos do $4^{0}$ ano em comparação aos demais. Essa circunstância remete à necessidade de intensificar o ensino frente às bases conceituais de LPP, no processo de formação desses profissionais, pois o conhecimento quanto ao processo de avaliação e classificação é indispensável ao tratamento adequado, por se tratar do ponto de partida para o sucesso terapêutico ${ }^{(15)}$. Há evidências crescentes que, para garantir a segurança do paciente no que se refere à LPP, a formação adequada do profissional sobre como realizar uma avaliação completa da pele, que inclua as técnicas de identificação de respostas ao branqueamento, calor local, edema e rigidez, é imprescindível para prevenir, classificar, diagnosticar e tratar LPP ${ }^{(9)}$. Por isso, torna-se demanda relevante e atual no contexto da assistência segura.

Nesse sentido, a avaliação e a classificação das LPP, pelos acadêmicos e futuros profissionais de enfermagem, são variáveis que se tornam imperativas para o rol de condutas assertivas, em especial, as relacionadas ao tratamento. Destaca-se que, no contexto da presente pesquisa, apenas a questão referente à classificação do estágio IV apresentou um percentual considerado satisfatório. Este resultado indica a necessidade de aprimoramento do conhecimento dos acadêmicos em relação às demais classificações das LPP.

No Irã, estudo que se propôs a identificar o conhecimento acerca do tema em duas faculdades de enfermagem realçou que os acadêmicos apresentaram maior índice de acerto nas questões relativas à avaliação da LPP $^{(22)}$. Essas diferenças podem ser justificadas por duas razões: pelo perfil da população estudada; e pelo tipo de método de ensino adotado pelas instituições. Este último impacta no nível de conhecimento adquirido pelos acadêmicos, da mesma forma que fortalece, ou não, a retenção do conteúdo apreendido pelos anos subsequentes, os quais contribuem para a adoção de ações e decisões que impactem diretamente nos métodos de prevenção de LPP, no conhecimento de sua ocorrência e em suas consequências. Da mesma forma, contribui para a utilização de estratégias de monitoramento e de adoção de indicadores de qualidade preconizados pelo protocolo nacional de prevenção de LPP. O objetivo deste protocolo é avaliar as mudanças direcionadas às 
melhorias no desempenho dos profissionais em relação às práticas seguras e ao impacto na redução da incidência desse agravo em todos os serviços de saúde do país ${ }^{(6,12)}$.

Na categoria 2 (Medidas Preventivas de LPP), no que se refere às questões relacionadas à avaliação de risco de LPP, das orientações que devem ser oferecidas ao paciente com mobilidade reduzida e seus familiares, e a educação continuada por meio de programas educacionais nas instituições, objetivando reduzir esses eventos, observou-se que esses itens foram os que apresentaram maior número de acertos entre os acadêmicos do último ano. Entretanto, os dados da presente pesquisa revelaram conhecimento insatisfatório em vários itens de prevenção, incluindo aqueles relacionados à inspeção diária da pele e ao uso de tecnologias para alívio e/ou redistribuição da pressão. Achados similares foram encontrados em Escola de Enfermagem da Índia, em que se observou que 48,9\% (n=41) dos acadêmicos relataram desconhecimento sobre as práticas de prevenção ${ }^{(20)}$.

As diretrizes internacionais e nacionais em prevenção de LPP e segurança do paciente enfatizam a avaliação estruturada, por meio de escala validada, na admissão do paciente. Deve contemplar a identificação de alterações significativas na condição de saúde do paciente, tais como atividade/mobilidade e condição da pele $e^{(6,9-10)}$. A pronta identificação da estratificação dos pacientes em risco para o desenvolvimento de LPP permite desenvolver e implementar um plano de prevenção baseado no risco individual e disponibilizar recursos institucionais para adoção de medidas imediatas ${ }^{(6)}$.

Ainda nessa categoria 2, notou-se que a média de acertos dos acadêmicos do $4^{\underline{0}}$ ano foi significativamente superior à média do $2^{\circ}$ ano $(\mathrm{p}=0,006)$, o que corrobora a investigação multicêntrica conduzida na Itália, a qual identificou que a elevada escolaridade dos acadêmicos de enfermagem estava significativamente relacionada aos maiores escores totais de conhecimento $(\mathrm{p}<0,001)^{(23)}$. É esperado que o conhecimento dos acadêmicos acerca do tema abordado deva apresentar-se de forma progressiva com a evolução dos anos, sendo obrigatória a atuação prática em diversos contextos de saúde, para a realização de estágios supervisionados. Some-se ainda as atividades extracurriculares e os treinamentos universitários, que contribuem para a aquisição e o aperfeiçoamento desse conhecimento, como condições essenciais para o desenvolvimento de atitudes, habilidades e competências ${ }^{(16)}$.

Entretanto, cabe destacar que, além do conhecimento insatisfatório em vários itens da categoria 2, os acadêmicos do $4^{\circ}$ ano citaram intervenções contraindicadas, como, por exemplo, massagear áreas hiperemiadas, com índice de erros de 69\%. Também mostraram desconhecer as recomendações para prevenção de LPP, ao não indicarem o uso de luvas d'água ou de ar para aliviar a pressão dos calcâneos ${ }^{(6,9)}$. Essa questão obteve o maior índice de erro $(83,3 \%)$, com diferença significativa para os demais anos $(\mathrm{p}=0,002)$.

É evidente o risco de dano que pode ser ocasionado ao paciente, em decorrência do conhecimento incipiente e certamente baseado na intuição e em experiências ultrapassadas, que podem limitar o cuidado seguro na prática clínica. Com isso, é imperativo implementar ações pedagógicas diversificadas sobre a avaliação, a classificação e a prevenção de LPP, com destaque para a articulação entre teoria e prática, a fim de alcançar resultados satisfatórios de conhecimento ${ }^{(15-16)}$. Considerando a importância da segurança do paciente e sua melhoria, parece ser essencial que a implementação das recomendações para a prevenção e manejo precoce de LPP dependem de uma forte liderança e do trabalho em equipe e precisam integrar os aspectos clínicos, educacionais e gerenciais ${ }^{(12)}$.

Vale salientar que abordar e incrementar ações sistemáticas sobre a temática da LPP tornam-se relevantes e apropriadas para suprir as lacunas de conhecimento encontradas. É importante que essas possam impactar significativamente no conhecimento dos acadêmicos, tais como experiências clínicas, para intensificar a capacidade pessoal e intelectual, e atividades que estimulem o aprendizado, sejam elas palestras, 
12

Conhecimento de acadêmicos de enfermagem sobre lesões por pressão: desafio para a segurança do paciente

cursos presenciais e on-line, casos clínicos, aulas práticas, pesquisas e cursos de extensão ${ }^{(16)}$.

Aliás, os dados desta pesquisa remetem ao entendimento da necessidade de superar o modelo tradicional de ensino, que não mais atende à demanda de formação dos profissionais de enfermagem. Nesse sentido, a prática baseada em evidências vem sendo adotada como estratégia de ensino e aprendizagem na prevenção de LPP $^{(24)}$. Também as simulações têm sido um recurso utilizado com o objetivo de aprimorar as habilidades e o conhecimento, promovendo discussões críticas que capacitem os acadêmicos de enfermagem para a realização da assistência segura ao paciente ${ }^{(25)}$.

Fica evidente, então, que é de extrema importância que os educadores apropriem-se de conhecimentos adequados sobre LPP, para O desenvolvimento de ensino teórico e prático de qualidade aos acadêmicos de enfermagem, pois esses, quando formados, precisarão de respaldo técnico-científico e de julgamento clínico para atuar na profissão com melhor desempenho e autonomia $^{(15)}$. Ademais, esses acadêmicos serão responsáveis por assegurar a continuidade dos núcleos de segurança do paciente, com a finalidade de manter um sistema de vigilância, monitoramento, prevenção e mitigação de eventos relacionados à assistência à saúde, especialmente das LPP. Deve também contribuir para a construção e implementação de materiais técnicos e educativos, como, por exemplo, cartazes sobre prevenção de LPP, com vistas a ofertar boas práticas em serviços de saúde, auxiliando na prevenção e minimização desse agravo e contribuindo para a segurança do paciente ${ }^{(12)}$.

Acredita-se que a articulação entre professores, preceptores e acadêmicos de enfermagem, diante da necessidade de alcançar conhecimento satisfatório para a promoção de práticas seguras, seja um desafio por duas razões: por um lado, torna-se elemento desafiador, pela necessidade de integrar o saber teórico à prática nos serviços de saúde; por outro lado, oportuniza elaborar estratégias de ensino e ações inovadoras, com vistas a proporcionar melhorias frente à avaliação, classificação e prevenção desse tipo de
EA pelos acadêmicos e, assim, reduzir os riscos associados ao cuidado prestado.

Entre as limitações reconhecidas destacam-se a não investigação do conhecimento dos professores referente à avaliação, à classificação e à prevenção de LPP e também o fato de a pesquisa ter sido conduzida em uma única instituição de ensino. Investigações futuras, com o foco em especial nos métodos utilizados para a condução do ensino no tema abordado e no conhecimento prévio dos professores de enfermagem em LPP, são cogentes para complementar a análise dos achados desta pesquisa.

\section{Conclusão}

O conhecimento dos acadêmicos de enfermagem referente à avaliação, classificação e às medidas preventivas de LPP foi considerado insatisfatório. Nota-se que a distribuição dos acertos entre os anos foi similar no quesito avaliação e classificação, enquanto que, para as medidas preventivas, os acadêmicos de enfermagem do $4^{\circ}$ ano apresentaram significativamente o maior número de acertos. Constata-se a necessidade de aprimorar e desenvolver novas estratégias de ensino-aprendizagem na instituição de ensino investigada, com a intenção de avançar progressivamente em melhorias na formação de profissionais enfermeiros.

Os dados encontrados reforçam a necessidade de criar ações institucionais voltadas ao processo de ensino-aprendizagem, como, por exemplo, ambientes simulados, jogos didáticos e desenvolvimento de algoritmos de inteligência artificial que auxiliem os acadêmicos de enfermagem a avaliar, identificar e prevenir os fatores de risco para LPP. A lacuna encontrada no conhecimento de LPP na formação acadêmica de enfermeiros acerca do tema abordado suscita a importância de intensificar a educação continuada nos serviços de saúde, para aperfeiçoar a atuação desse profissional na prática clínica.

A adoção dessas estratégias, diante dos resultados encontrados na presente pesquisa, contribuirá potencialmente para o elenco de decisões atinentes para que gestores, profissionais da 
prática e da academia possam aprimorar o conhecimento sobre a temática investigada. Dessa forma, será possível atender aos preceitos do PNSP e às resoluções do conselho de classe profissional, no que se refere ao cuidado à pessoa com lesão de pele, visando solidificar bases conceituais e adotar medidas preventivas para a redução de LPP, promovendo a segurança do paciente em serviços públicos e privados do país.

\section{Colaborações:}

1 - concepção, projeto, análise e interpretação dos dados: Ana Flávia Furtado, Larissa Marcondes, Bruna Eloise Lenhani e Josemar Batista;

2 - redação do artigo e revisão crítica relevante do conteúdo intelectual: Ana Flávia Furtado, Larissa Marcondes, Bruna Eloise Lenhani e Josemar Batista;

3 - aprovação final da versão a ser publicada: Ana Flávia Furtado, Larissa Marcondes, Bruna Eloise Lenhani e Josemar Batista.

\section{Referências}

1. Conselho Federal de Enfermagem. Resolução n. 567, de 29 de janeiro de 2018. Aprova o Regulamento da atuação da Equipe de Enfermagem no Cuidado aos pacientes com feridas [Internet]. Brasília (DF); 2018 [cited 2019 Feb 28]. Available from: http://www.cofen.gov.br/ resolucao-cofen-no-567-2018_60340.html

2. Beeckman D, Van Damme N, Meyer D, Van den Bussche K. Pressure Ulcers. In: Roller-Wirnsberger R, Singler K, Polidori M, editores. Learning Geriatric Medicine. Practical Issues in Geriatrics [Internet]. Cham (SWI): Springer; 2018. p. 179-89. DOI: https://doi. org/10.1007/978-3-319-61997-2_19

3. Constante SAR, Oliveira VC. Lesão por pressão: uma revisão de literatura. Rev Psicol Saúde Debate. 2018;4(2):95-114. DOI: https://doi. org/10.22289/2446-922X.V4N2A6

4. Moraes JT, Borges EL, Lisboa CR, Cordeiro DCO, Geralda Rosa E, Rocha NA. Conceito e classificação de lesão por pressão: atualização do National Pressure Ulcer Advisory Panel. Rev enferm Cent-Oeste Min. 2016;6(2):2292-306. DOI: http://dx.doi.org/10.19175/recom.v6i2.1423
5. National Pressure Ulcer Advisory Panel. NUAP Pressure injury stages [Internet]. Westford (USA); 2016 [cited 2019 Nov 3]. Available from: https:// cdn.ymaws.com/npuap.site-ym.com/resource/ resmgr/npuap_pressure_injury_stages.pdf

6. Brasil. Ministério da Saúde. Portaria n. 1.377, de 9 de julho de 2013. Aprova os protocolos de segurança do paciente: anexo 2, protocolo para prevenção de úlcera por pressão [Internet]. Brasília (DF); 2013 [cited 2019 Nov 17]. Available from: https://www20.anvisa.gov.br/ segurancadopaciente/index.php/publicacoes/ item/ulcera-por-pressao

7. Liu Y, Wu X, Ma Y, Li Z, Cao J, Liu G, et al. The prevalence, incidence, and associated factors of pressure injuries among immobile inpatients: A multicentre, cross-sectional, exploratory descriptive study in China. Int Wound J. 2019;16(2):459-66. DOI: https://doi.org/10.1111/iwj.13054

8. Brasil. Agência Nacional da Vigilância Sanitária. Segurança do paciente: relatórios dos estados - eventos adversos - arquivos [Internet]. Brasília (DF); 2019 [cited 2019 Jul 17]. Available from: https://www20.anvisa.gov.br/ segurancadopaciente/index.php/publicacoes/ category/relatorios-dos-estados

9. National Pressure Ulcer Advisory Panel. European Pressure Ulcer Advisory Panel. Pan Pacific Pressure Injury Alliance. Prevention and Treatment of Pressure Ulcers: Quick Reference Guide [Internet]. Cambridge Media: Osborne Park, Western Australia; 2014 [cited 2019 Nov 3]. Available from: http://www.epuap.org/wpcontent/uploads/2016/10/quick-reference-guidedigital-npuap-epuap-pppia-jan2016.pdf

10. National Institute for Health and Care Excellence. Pressure ulcers: prevention and management [Internet]. United Kingdom; 2014 [cited 2019 Nov 17]. Available from: https://www.nice.org.uk/ guidance/cg179

11. Brasil. Ministério da Saúde. Portaria n. 529, de $1^{\circ}$ de abril de 2013. Institui o Programa Nacional de Segurança do Paciente (PNSP) [Internet]. Brasília (DF); 2013 [cited 2019 Feb 28]. Available from: http://bvsms.saude.gov.br/bvs/saudelegis/ gm/2013/prt0529_01_04_2013.html

12. Brasil. Agência Nacional de Vigilância Sanitária. Nota Técnica GVIMS/GGTES nº 03/2017. Práticas seguras para prevenção de Lesão por Pressão em serviços de saúde [Internet]. Brasília (DF); 2017 [cited 2019 Nov 17]. Available from: https://www20. 
anvisa.gov.br/segurancadopaciente/index.php/ alertas/item/nota-tecnica-gvims-ggtes-03-2017

13. Pieper B, Mott M. Nurses' knowledge of pressure ulcer prevention, stating, and description. Adv Wound Care [Internet]. 1995 [cited 2019 Jun 25];8(3):34-8.Available from: https://www.ncbi.nlm. nih.gov/pubmed/7795877

14. Fernandes LM, Caliri MHL, Haas VJ. The effect of educative interventions on the pressure ulcer prevention knowledge of nursing professionals. Acta Paul Enferm. 2008;21(2):305-11. DOI: http:// dx.doi.org/10.1590/S0103-21002008000200012

15. Baratieri T, Sangaleti CT, Trincaus MG. Conhecimento de acadêmicos de enfermagem sobre avaliação e tratamento de feridas. Rev enferm atenção saúde [Internet]. 2015 [cited 2019 Sep 22];4(1):2-15. Available from: http://seer.uftm. edu.br/revistaeletronica/index.php/enfer/article/ view/1259/1130

16. Ribeiro AMN, Ribeiro EKC, Ferreira MTA, Souza JERB, Silva AAS, Baldoino LS. The knowledge of nursing undergraduatestudents about pressure lesions. Rev Rene. 2019;20:e41016. DOI:https://doi.org/10.15253/2175-6783.20192041016

17. Eberle CC, Silva APSS. Compreensão de estudantes de enfermagem sobre a segurança do paciente. Rev baiana enferm. 2016 out/dez;30(4):1-9. DOI: http://dx.doi.org/10.18471/rbe.v30i4.21701

18. Galvão NS, Serique MAB, Santos VLCG, Nogueira PC. Knowledge of the nursing team on pressure ulcer prevention. Rev bras enferm. 2017;70(2):294-300. DOI: http://dx.doi. org/10.1590/0034-7167-2016-0063

19. Cardoso DS, Carvalho FMO, Rocha GB, Mendes JR, Cardoso SB, Rocha FCV. The Nurses' knowledge with regards to both classification and prevention of pressure injury. J res: fundam care. 2019;11(3):560-6. DOI: $\quad$ http://dx.doi.org/10.9789/2175-5361.2019. v11i3.560-566
20. Bollineni NJ. Knowledge and Practices of Student Nurses of NRI College of Nursing in Application of SSKIN towards Prevention of Pressure Ulcers in NRI Hospital, Chinakakani, Mangalagiri, Guntur (Dt). Asian J Nurs Educ Res. 2019;9(1):17-22. DOI: http://dx.doi.org/10.5958/2349-2996.2019.00004.1

21. Lopes CM, Andrade EMLR, Luz MHBA. Conhecimento de graduandos de enfermagem sobre úlcera por pressão. Enferm foco. 2015;6(1/4):24-30. DOI: https://doi.org/10.21675/2357707X.2015.v6.n1/4.572

22. Rafiei H, Mehralian H, Abdar ME, Madadkar T. Pressure ulcers: how much do nursing students really know? Br J Nurs. 2015 Mar;24(6):S12-S14-7. DOI: https://doi.org/10.12968/bjon.2015.24.Sup6. S12

23. Simonetti V, Comparcini D, Flacco ME, Giovanni P, Cicolini G. Nursing students' knowledge and attitude on pressure ulcer prevention evidencebased guidelines: a multicenter cross-sectional study. Nurse Educ Today. 2015 Apr;35(4):573-9. DOI: https://doi.org/10.1016/j.nedt.2014.12.020

24. Volpato MP, Caliri MHL, Galdino MJQ, Martins JT. Percepción de estudiantes de enfermería sobre el aprendizaje basado en evidencias. Investig enferm. 2018;20(1):9-11. DOI: https://doi.org/10.11144/ Javeriana.ie20-1.peea

25. Guinea S, Andersen P, Reid-Searl K, Levett-Jones T, Dwyer T, Heaton L, et al. Simulation-based learning for patient safety: The development of the Tag Team Patient Safety Simulation methodology for nursing education. Collegian. 2019;26(3):392-8. DOI: https://doi. org/10.1016/j.colegn.2018.09.008

Recebido: 5 de novembro de 2019

Aprovado: 20 de novembro de 2019

Publicado: 11 de março de 2020

A Revista Baiana de Enfermagem utiliza a Licença Creative Commons - Atribuição-NãoComercial 4.0 Internacional. https://creativecommons.org/licenses/by-nc/4.0/

Este artigo é de acesso aberto distribuído sob os termos da Licença Creative Commons (CC BY-NC).

Esta licença permite que outros remixem, adaptem e criem a partir do seu trabalho para fins não comerciais. Embora os novos trabalhos tenham de lhe atribuir o devido crédito e não possam ser usados para fins comerciais, os usuários não têm de licenciar esses trabalhos derivados sob os mesmos termos. 